\title{
Mechanosensitive Pathways Involved in Cardiovascular Development and Homeostasis in Zebrafish
}

\author{
Rongsong $\mathrm{Li}^{\mathrm{a}}$ Kyung In Baek ${ }^{\mathrm{b}}$ Chih-Chiang Chang ${ }^{\mathrm{b}}$ Bill Zhou ${ }^{\mathrm{c}}$ \\ Tzung K. Hsiai ${ }^{b, d}$ \\ ${ }^{a}$ College of Health Sciences and Environmental Engineering, Shenzhen Technology University, Shenzhen, China; \\ ${ }^{b}$ Department of Bioengineering, University of California, Los Angeles, CA, USA; \\ 'Department of Radiology, University of California, Los Angeles, CA, USA; \\ ${ }^{\mathrm{d}}$ Department of Medicine (Cardiology) and Bioengineering, University of California, Los Angeles, CA, USA
}

\section{Keywords}

Zebrafish · Shear stress - Mechanosensitive pathways ·

Mechanotransduction · Cardiovascular development

\begin{abstract}
Cardiovascular diseases such as coronary heart disease, myocardial infarction, and cardiac arrhythmia are the leading causes of morbidity and mortality in developed countries and are steadily increasing in developing countries. Fundamental mechanistic studies at the molecular, cellular, and animal model levels are critical for the diagnosis and treatment of these diseases. Despite being phylogenetically distant from humans, zebrafish share remarkable similarity in the genetics and electrophysiology of the cardiovascular system. In the last 2 decades, the development and deployment of innovative genetic manipulation techniques greatly facilitated the application of zebrafish as an animal model for studying basic biology and diseases. Hemodynamic shear stress is intimately involved in vascular development and homeostasis. The critical mechanosensitive signaling pathways in cardiovascular development and pathophysiology previously studied in mammals have been recapitulated in zebrafish. In this short article, we reviewed recent
\end{abstract}

\section{KARGER}

(c) 2019 S. Karger AG, Basel

E-Mail karger@karger.com

www.karger.com/jvr knowledge about the role of mechanosensitive pathways such as Notch, PKCE/PFKFB3, and Wnt/Ang2 in cardiovascular development and homeostasis from studies in the zebrafish model.

(c) 2019 S. Karger AG, Basel

\section{Introduction}

Cardiovascular diseases are the leading cause of death and increasingly becoming a worldwide burden [1]. While the advancement of biomedical research promises newer, faster, and cheaper diagnoses and therapies, the fundamentals of discovery remain deeply rooted in elucidating the pathogenic mechanisms at the molecular and cellular levels, especially with the advent of personalized medicine. Using animal models, gene functions and signaling pathways can be linked to normal development and pathophysiology, allowing for the identification and

Rongsong Li

College of Health Sciences and Environmental Engineering Shenzhen Technology University

3002 Lantian Road, Pingshan District

Shenzhen, Guangdon 518118 (China)

E-Mail lirongsong@sztu.edu.cn

Tzung K. Hsiai

Department of Medicine (Cardiology) and Bioengineering

University of California

650 Charles Young Drive

Los Angeles, CA 90095 (USA)

E-Mail thsiai@mednet.ucla.edu 
validation of pharmacological targets for novel therapeutics.

The zebrafish has been utilized as an important developmental model because it shares a largely conserved physiology and anatomy with that of mammals. Its unique transparency at the larval stage enables direct observation of organ development, including the cardiovascular system [2-5]. More recently, the role of zebrafish as human disease models has been expanded to include the adult zebrafish due to a genome that bears similarity to humans $[4,6,7]$ and the relative ease of genetic manipulation utilizing the latest genomic engineering approaches [8-10]. Simple husbandry and rapid development enable a range of large-scale phenotypic screening [3, 4, 7]. Furthermore, the zebrafish demonstrates impressive regenerative capacity, such as vascular repair and heart regeneration, that scientists hope to unlock for therapeutic applications in humans.

\section{Results and Discussion}

\section{Mechanosensors and Mechanotransduction}

Hemodynamic shear stress, the frictional force from blood flow acting on vascular endothelial cells (ECs), plays a crucial role in vascular development and homeostasis. Many membrane-associated structures and molecules have been identified as sensors of shear stress to convert mechanical force into biochemical signals. These mechanosensors fall into 3 categories based on their localization on the cell surface: luminal, junctional, and basal [11]. On the luminal surface, plasma membrane microdomain structures such as cilia, glycocalyx, and caveolae are able to transduce mechanical stimuli [12]. Other luminal mechanosensors include G-protein-coupled receptors such as sphingosine 1-phosphate receptor 1 and the bradykinin B2 receptor, and ion channels [13-15]. Junctional molecules PECAM-1 and VE-cadherin, mainly located on the lateral surface, couple together with vascular endothelial growth factor (VEGF) receptors (VEGFRs) to form mechanosensory complexes, which may serve as the primary mechanosensors of shear force in EC $[11,16]$. Integrins on the basal surface of EC connect extracellular matrix (ECM) and actin cytoskeleton, making them likely candidates of mechanosensors. Although it is still debatable whether integrins directly sense shear stress, a large body of studies support their role as integrators of shear-dependent signaling pathways [1719]. The many mechanosensing molecules of ECs reported in the literature can sense shear forces either directly or indirectly, with some having both sensing and adaptor functions. Cross talk among these mechanosensors may be important to translating physical force into biochemical signaling.

Downstream of mechanosensors, small GTPases act as an important set of signaling molecules that transduce shear stress-mediated signaling. Members of the Rho family of GTPases are highly sensitive to both spatial and temporal regulation by shear stress [20]. Shear stress transiently regulates the activities of RhoA and Rac, and the proper spatiotemporal activation of both Rho and Rac is required for aligning ECs in the direction of flow [17, 21]. In ECs subjected to shear stress, Cdc 42 translocates to the EC membrane and directs the orientation of the microtubule organizing center (MTOC) [22, 23]. An increasing number of studies have demonstrated that distinct signaling pathways activated by shear stress can be coordinated via activation of small GTPases [19, 20, 24].

Multiple mechanosensitive signaling pathways downstream of mechanosensors and mechanotransducers act to modulate endothelial function [25]. Functional status of ECs is central to cardiovascular homeostasis and physiology. While many in vitro and in vivo models have been utilized to investigate mechanotransduction, the assignment of specific mechano-signaling pathways to cardiovascular physiology and pathology remains a challenge. Zebrafish is emerging as an excellent model to meet the challenge.

\section{Mechanosensitive Pathways in Vascular Development} and Regeneration in the Zebrafish

Hemodynamic fluid shear stress provides biomechanical cues for the differentiation of stem cells $[26,27]$ and mesenchymal progenitors [28] into vascular ECs [26, 29, 30]. Taking advantage of the zebrafish model system, our lab and others have demonstrated that shear stress activates mechanosensitive pathways such as Notch, protein kinase $\mathrm{C}$ isoform $\varepsilon$ (PKC $\varepsilon$ )/6-phosphofructo-2-kinase/ fructose-2,6-biphosphatase 3 (PFKFB3), and Wnt/angiopoietin-2 (Ang2) pathways that are implicated in vascular development and regeneration (Fig. 1).

\section{Notch Signaling in Vascular Regeneration}

It is well established that Notch signaling is essential for vascular morphogenesis. Ablation of Notch 1 causes developmental retardation and results in embryonic lethality [31]. Missense mutation in the Notch3 gene causes the development of the degenerative vascular disease known as CADASIL (cerebral autosomal-dominant arteriopathy with subcortical infarcts and leukoencephalopa- 


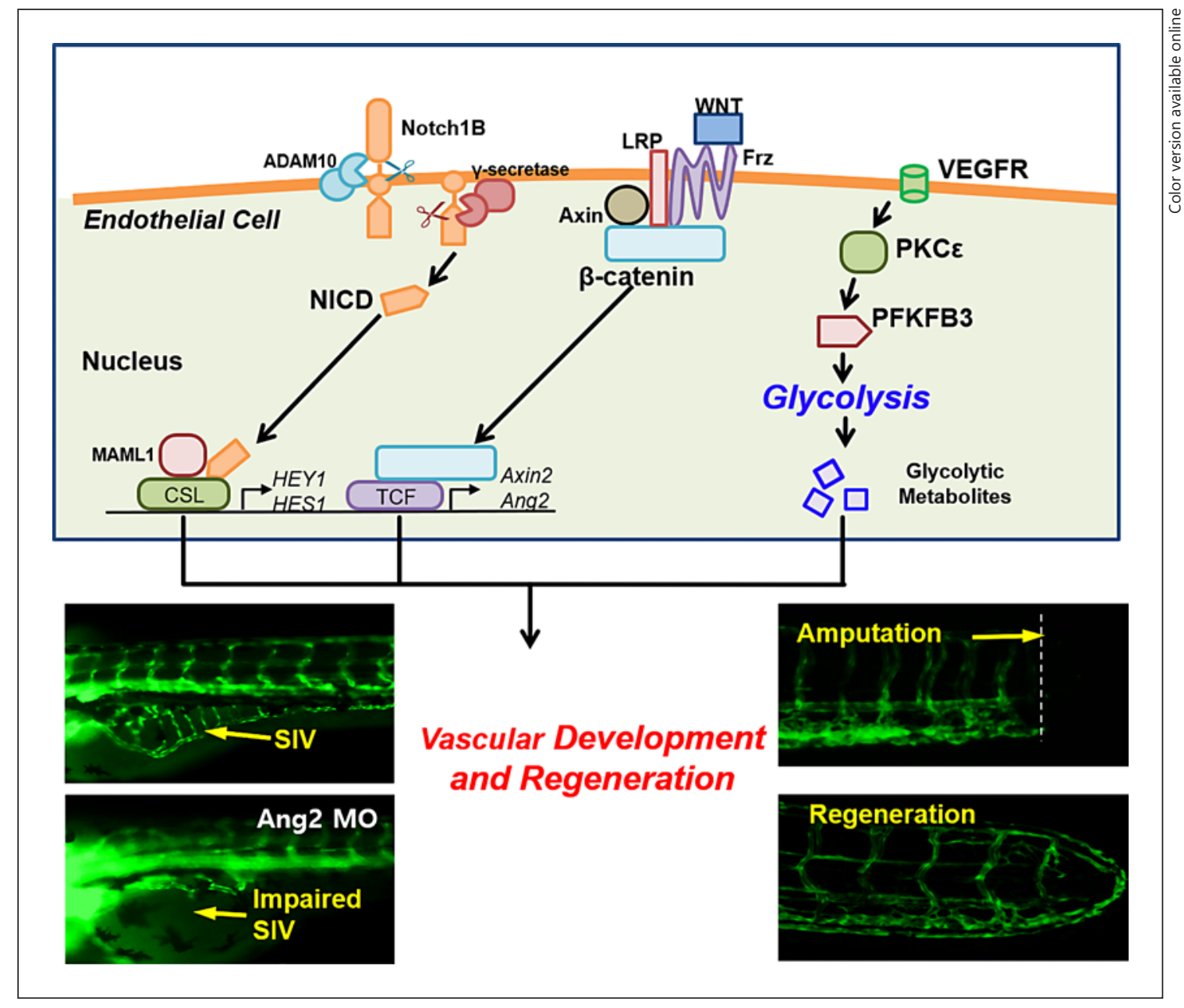

Fig. 1. Mechanosensitive signal pathways underlying zebrafish vascular development and regeneration. Hemodynamic shear stress activates multiple mechanical and metabolic pathways including Notch, Wnt/Ang2, and $\mathrm{PKC \varepsilon /PFKFB3}$ pathways to modulate vascular development and regeneration in zebrafish.

thy) [32]. Dysregulated Notch activity induces failure of vascular specification and results in abnormal endothelial proliferation, leading to the formation of a hyperplastic vascular network $[31,33]$. The role of Notch signaling in vascular homeostasis was only recently elucidated. Mack et al. [34] reported that endothelial Notch1 is shear stress sensitive and necessary for the maintenance of junctional integrity, cell elongation, and suppression of proliferation. In embryonic stem cell-derived murine endothelial progenitor cells, unidirectional laminar shear stress activates the VEGF-Notch pathway to drive the expression of the arterial endothelial marker EphrinB2 (EFNB2) and to downregulate the venous endothelial marker EphrinB4 [35]. Use of embryonic zebrafish allows for genetic manipulations of blood viscosity to alter the level of endothelial wall shear stress [36] and subsequently Notch signaling in vivo. Our observations in an embryonic zebrafish tail amputation model of vascular injury and repair support the notion that shear-sensitive Notch signaling plays an important role in vascular regeneration [37]. While control zebrafish developed vascular regeneration between the dorsal aorta and the dorsal longitudinal anastomotic vessel 3 days post-tail amputation (dpa), inhibition of Notch signaling such as using GI254023X, a pharmacological inhibitor of A disintegrin and metalloproteinase domain-containing protein 10 (ADAM10), to prevent proteolytic cleavage of Notch extracellular domain (NECD) and injecting dominant negative (DN)-Notch1b mRNA resulted in impaired vascular regeneration. As a corollary, injection of NICD mRNA to 
promote Notch signaling restored vascular regeneration impaired by GI254023X and DN-Notch $1 b$ mRNAs [37].

PKCE/PFKFB3 Pathway in Vascular Regeneration

$\mathrm{PKC} \varepsilon$ is a family of serine and threonine kinases involved in cell differentiation, proliferation, and migration [38-40]. PKCe is abundantly expressed in EC and is closely associated with a series of pathways involved in angiogenesis and vascular formation $[41,42]$. The flowresponsive VEGFR-endothelial nitric oxide synthase pathway drives the expression of PKCE to maintain endothelial homeostasis and lumen formation [43-45]. Shearmediated nitric oxide production $[46,47]$ further modulates $\mathrm{PKC} \varepsilon$ expression, which in turn attenuates mitochondrial reactive oxygen species following ischemia or reperfusion injury [48-52]. ECs are highly glycolytic [53], and endothelial glycolysis is mechanoresponsive [54]. When switching from quiescence to the proliferating state, ECs increase the level of glycolytic flux while glycolytic enzymes including PFKFB3 localize to lamellipodia [55]. As a rate-limiting enzyme and critical regulator of glycolysis, PFKFB3 regulates lamellipodia and filopodia extension for vessel formation. Our recent observation with embryonic zebrafish suggested that intravascular fluid shear stress modulates PKCe-PFKFB3 to promote vascular regeneration after injury. In the transgenic $\mathrm{Tg}(f l k-1:$ GFP) zebrafish tail amputation model, the control zebrafish developed robust vascular regeneration 3 dpa while shear stress reduction via Gata la morpholino oligonucleotide $(\mathrm{MO})$ injection delayed vascular regeneration from 3 to 5 dpa [56]. Knockdown of cardiac troponin T2 (tnnt2a) with MO as a means of arresting myocardial contractility and blood flow also resulted in impairment of vascular regeneration. Injection of the PKCE mRNA overrides the effect of Gatala MO, supporting the hypothesis that flow-driven vascular regeneration is at least partially mediated by $\mathrm{PKC} \varepsilon$ [56].

The Wnt/Ang2 Pathway in Vascular Development and Regeneration

Extensive studies have demonstrated the role of Ang1 and Ang2 in vascular development. Shear stress-mediated Ang2 in mature vascular endothelium was recently reported to play a role in tubulogenesis [57] and conferring atheroprotection [58]. While Ang1 is constitutively released by perivascular cells, Ang2 is expressed in ECs released from the Weibel-Palade bodies upon signal cues [59, 60]. Ang2, like Ang1, binds to endothelial-specific receptor tyrosine kinase 2 (TIE2) and acts as a negative regulator of Ang1/TIE2 signaling to promote angiogen- esis [61]. Earlier studies demonstrated that Ang2 could be released by mechanical force such as endothelial stretch that occurs during hypertension [62]. Our recent work as well as work from Dr. Jo's lab both demonstrated Ang2 as a mechanosensitive gene involved in shear stress-mediated tubule formation and migration of ECs [57, 63]. In the mouse artery occlusion model, femoral artery ligation caused disturbed flow to stimulate Ang2 expression and arteriogenesis in mice [64].

Canonical Wnt/ $\beta$-catenin signaling is a pivotal pathway regulating development, cell proliferation, and migration [65]. We further demonstrated that shear stressstimulated Ang2 expression is mediated by the canonical Wnt signaling pathway. While a Wnt agonist, Wnt3a, promoted Ang2 expression, inhibition of Wnt signaling with Dickkopfs-1 (Dkk-1) or IWR-1 attenuated EC migration and tube formation. In the heat shock-inducible DKK-1 transgenic Tg (hsp70l: Dkk1-GFP) zebrafish embryos, Ang2b (zebrafish Ang2) expression was downregulated upon heat shock. Ang2 MO injection into transgenic $T g(k d r l: G F P)$ zebrafish to downregulate Ang2 resulted in impaired development of subintestinal vessels $72 \mathrm{~h}$ after fertilization. Inhibition of Wnt signaling also impaired subintestinal vessel development that is rescued with Ang2 mRNA. In the zebrafish tail amputation model, inhibition of Wnt signaling retarded and reduced the rate of vascular regeneration that is also rescued by Ang2 mRNA [63]. These findings support the notion that the mechanosensitive Wnt/Ang2 pathway modulates vascular development and regeneration.

Hemodynamic Fluid Force on Cardiac Development via Mechanosensitive Notch Signaling in Zebrafish

In addition to transcription factors involved in cardiogenic differentiation, hemodynamic fluid forces also play an essential role in cardiogenesis [36, 66-71]. Occlusion of flow at either the cardiac inflow or outflow tracts resulted in hearts with an abnormal third chamber, diminished looping, and impaired valve formation [36]. During heart development, the myocardium differentiates into 2 layers: an outer compact zone and an inner trabeculated zone. The trabeculae form a network of branching outgrowths from the myocardial wall [72]. Both trabeculation and compaction are essential for normal cardiac contractile function. The reduction in trabeculation is associated with deficiency in the ventricular compact zone (hypoplastic wall) that commonly leads to embryonic heart failure and early embryonic lethality. On the other hand, hypertrabeculation is closely associated with left ventricular noncompaction cardiomyopathy [73], the 
third most common cardiomyopathy in the pediatric population after dilated and hypertrophic cardiomyopathies [74].

Cardiac trabeculation is a crucial morphogenetic process by which clusters of ventricular cardiomyocytes extrude and expand into the cardiac jelly to form sheet-like projections to enhance cardiac contractility and intraventricular conduction. Previous studies support the critical role of Notch signaling in heart development, including trabeculation $[75,76]$. Notch 1 has recently been reported as a mechanosensor in ECs [34], and Notch1 becomes sensitized to metalloprotease cleavage under shear stress [77]. Shear stress activates Notch signaling in both human and zebrafish endocardium [78]. In murine embryonic stem cell-derived vascular ECs, shear stress induces time-dependent Notch signaling [35]. To elucidate the role and mechanism of hemodynamic forces on trabeculation via notch signaling, Lee et al. [78] took advantage of genetic manipulation in zebrafish and lowered hemodynamic shear forces via: (1) microinjection of gata 1a MO at cell stages 1-4 to reduce hematopoiesis and viscosity by $90 \%[79,80]$, (2) microinjection of tnnt2a MO to arrest cardiomyocyte contraction in embryos [81, 82], and (3) genetic mutation of the weak atri$u^{m 58}$ (wea) mutant to inhibit atrial contraction $[72,83]$. Lowered hemodynamic forces correlated with the inhibition of Notch signaling and trabeculation. Utilizing the $T g(f l k-1: m$ Cherry, tp 1:gfp) fish line, shear stress-activated Notch signaling is confirmed to be localized to the endocardium. Endocardial activation of Notch signaling is required for trabeculation. The zebrafish Cloche $\left(c l o{ }^{\mathrm{m} 39 / \mathrm{lm} 2-\mathrm{GF}}\right)$ mutant, in which the endothelial line is abolished, developed small and thin ventricles $[84,85]$ along with reduced expression of cardiac Notch ligands, receptors, and target genes when compared to the wild type [78]. Notch activation in the endocardium results in the transcription of EphrinB2, which in turn upregulates neuregulin1 (Nrg1) [86]. As a secreted factor, Nrg1 signals to the adjacent cardiomyocytes to promote trabeculation. Disruption of Nrg1 expression after ischemic insult impairs cardiac contractility [87], whereas Nrg1 preconditioning confers cardiac protection from ischemic injury [88]. In parallel, Notch activity in the endocardium activates bone morphogenic protein 10 (BMP10) expression in the adjacent myocytes to promote proliferation [86]. Unlike mouse development, Nrg1/ErbB2 signaling contributes to both proliferation and differentiation of cardiomyocytes for trabeculation in zebrafish [89]. The advantages of the zebrafish model have enabled researchers to establish the essential role of mechanosen-

Cardiovascular Mechanotransduction in Zebrafish sitive Notch signaling in promoting cardiac trabeculation involving EphrinB2, Nrg1, and ErbB2 (Fig. 2) [86, $89,90]$.

\section{Regulation of Metabolic Pathways by Mechanical Forces}

Metabolomic analyses have led to the discovery of new metabolic biomarkers and therapeutic targets such as spermine for acute stroke, cinnamoylglycine, nicotinamide, and cysteine-glutathione disulfide for renal cancer, and 3-hydroxykynurenine and oxidized glutathione for Parkinson disease [91-93]. Hemodynamic forces have been shown to modulate mammalian metabolic pathways [94-96] to maintain vascular homeostasis. Elucidating flow-mediated metabolomic changes provides an entry point to uncovering metabolites involved in endothelial homeostasis [97, 98], migration [99], vascular development [53], and physical activity [100].

Endothelial glycolysis has been shown to be mechanoresponsive as well [54]. Rather than relying on oxidative metabolism for mitochondrial respiration, ECs generate over $80 \%$ of their adenosine triphosphate from the glycolytic pathway [101]. A recent study reports that laminar shear stress activates Krüppel-like factor 2 (KLF2) to modulate PFKFB3-mediated glycolysis, mitigating angiogenesis, and vessel sprouting [102]. On the other hand, flow-sensitive VEGFR signaling upregulates PFKFB3driven glycolysis $[53,55]$. We have recently demonstrated that shear stress promotes vascular regeneration and repair in zebrafish through VEGFR/PKCe/PFKFB3 signaling, and this effect is at least partially attributed to elevated glycolysis. Shear stress modulates a number of metabolites (Fig. 3a), including the elevation of the glycolytic metabolite dihydroxyacetone (DHA, $\left.\mathrm{C}_{3} \mathrm{H}_{6} \mathrm{O}_{3}\right)$, which is dependent on flow-sensitive PKCe (Fig. 3b). DHA partially rescued tube formation in human aortic ECs and impaired vascular regeneration and repair in zebrafish upon PKCe knockdown (Fig. 3c, d). Hemodynamic shear stress also modulates stearoyl-CoA desaturase-1 (SCD1) expression via PPAR $\gamma$ in ECs, a major pathway regulating lipid metabolism [103]. Metabolomic analysis in combination with genetic manipulation of hemodynamic shear force in zebrafish would greatly improve the investigation of in-depth mechanisms of mechanotransduction in vascular development and pathophysiology.

\section{Interaction and Synergy of Mechanosensitive}

Pathways

Multiple mechanosensitive pathways such as Notch, Wnt/Ang2, and PKCE/PFKFB3 signaling have been 


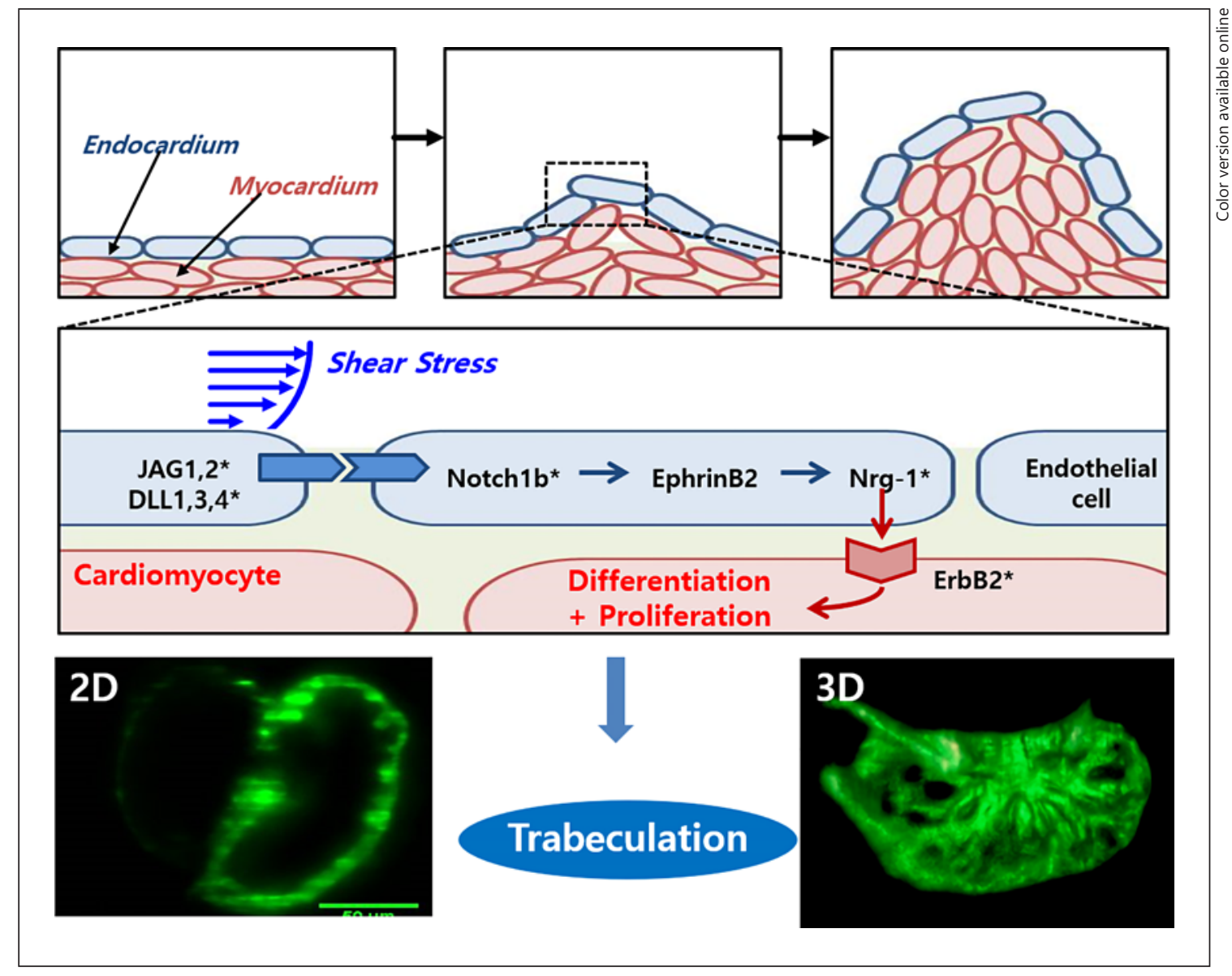

Fig. 2. Shear stress activation of Notch signaling promotes trabeculation in zebrafish. Shear stress activates Notch signaling in endocardial cells to upregulate the expression of Nrg1 via EphrinB2. Nrg1 promotes cardiomyocyte proliferation and differentiation for trabeculation.

shown to play important roles in vascular development and regeneration in the zebrafish model. Nakajima et al. [104] recently developed a zebrafish line to model blood flow and monitor the spatiotemporal localization and transcriptional activity of yes-associated protein (YAP) in ECs of living zebrafish. They revealed that blood flow regulates localization and activity of YAP, demonstrating flow-sensitive endothelial YAP is essential in vessel maintenance. It has recently been reported that flowsensitive SCD-1 generates palmitoleic acid. SCD1 activity modulates protein pamitoylation of TEA domain family (TEAD) members, the YAP/ transcriptional coactivator with PDZ-binding motif (TAZ) coactivator [105]. Furthermore, YAP regulates EC contact-mediated expression of Ang2 [106]. In cancer cells, SCD1 regulates YAP/TAZ activity via modulating $\mathrm{Wnt}$ signaling. Thus, mechanosensitive pathways are closely connected and interact with each other. The ability for easy genetic manipulation in zebrafish makes it an excellent model to study the interactions of mechanical force-activated signal pathways in cardiovascular development and homeostasis.

\section{Mechanosensitive Pathways of Different Mechanical} Forces in Cardiac Morphogenesis

In response to hemodynamic shear forces, myocardial ridges and grooves develop in a wave-like trabecular network in alignment with the direction of the shear stress across the atrioventricular valves [107]. Both trabeculation and compaction are essential for contractile function during cardiac development [72]. We demonstrated that hemodynamic shear forces modulate the initiation of cardiac trabeculation in zebrafish via endocardial NotchNrg1 signaling, which activates myocardial ErbB2 signal- 


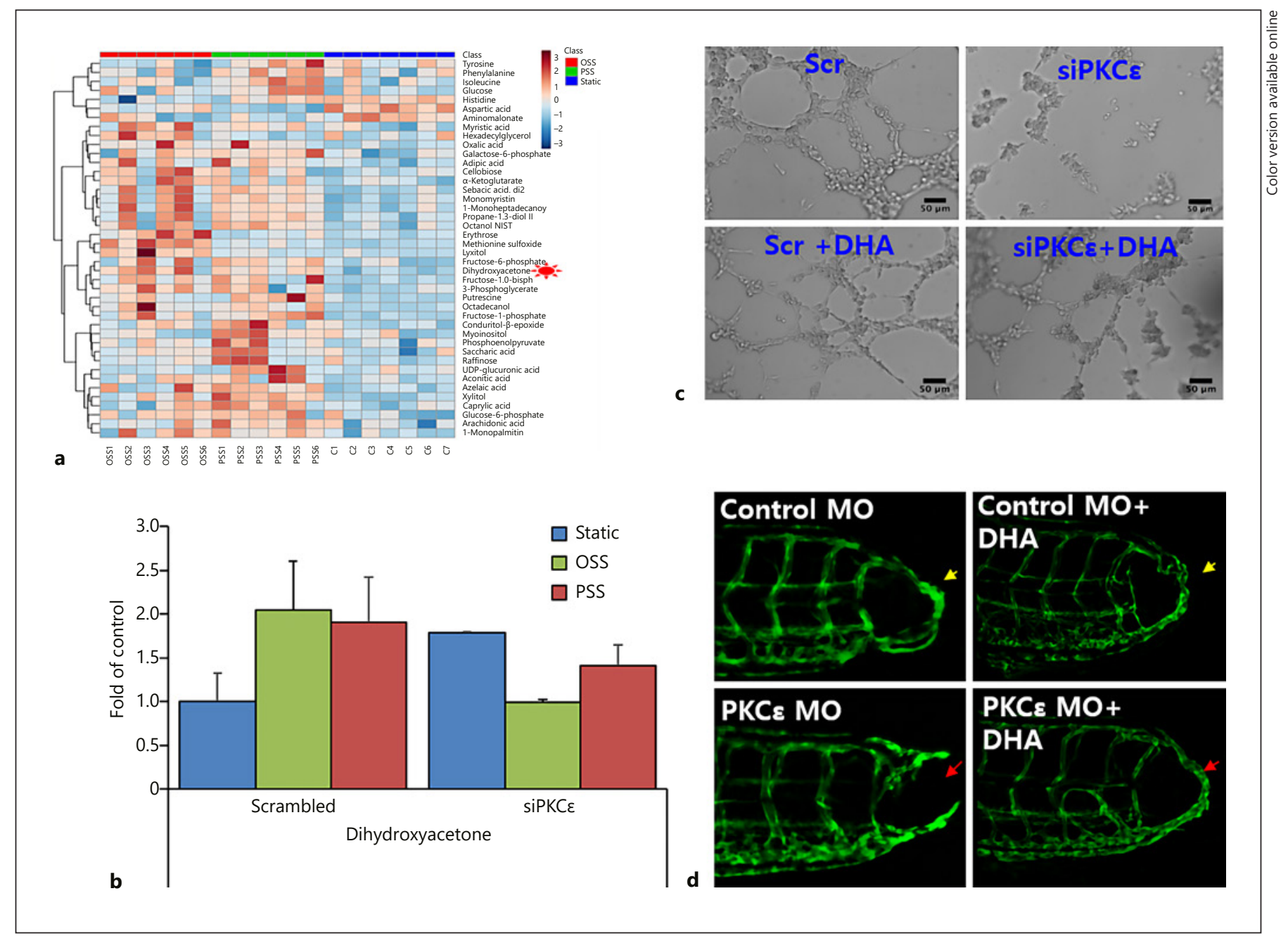

Fig. 3. The glycolytic metabolite dihydroxyacetone (DHA) promotes vascular regeneration/repair. a Pulsatile shear stress (PSS) and oscillatory shear stress (OSS) modulate a number of metabolites in human aortic endothelial cells (HAEC) including DHA. b The level of DHA is mechanosensitive dependent on PKCe. c DHA is able to rescue siPKCE-attenuated tube formation in HAEC. d DHA rescues $P K C \varepsilon$ MO-impaired vascular regeneration/repair in zebrafish. Scr/scrambled, scrambled control siRNA; siPKCe, PKCe siRNA; MO, morpholino oligonucleotide. Reprinted with permission from Antioxidants \& Redox Signaling [56].

ing to promote cardiomyocyte proliferation and differentiation to generate contractile force, which in turn activates Notch signaling $[89,108]$. Recently, Han et al. [109] reported that myocardial Notch signaling autonomously inhibits ErbB2 signaling to attenuate trabeculation in zebrafish. The different effects of hemodynamic shear force-induced endocardial Notch-Nrg1 signaling and myocardial contractile force-induced myocardial Notch signaling to inhibit ErbB2 may modulate the pattern formation of ridges and grooves during cardiac trabeculation [110].

Cardiovascular Mechanotransduction in Zebrafish

\section{Perspective and Conclusion}

The advancement and application of a diverse array of reverse genetic manipulations in zebrafish allow targeted investigation of a gene of interest in a specific pathway by increasing, reducing, or silencing its expression. An important and helpful resource for reverse genetic investigation is the zebrafish mutant project that provides a growing list of fish lines with a defined mutation in a specific gene [111]. For example, Lee et al. [78] demonstrated the role of shear stress-mediated notch signaling in trabecu- 
lation by taking advantage of the wea and clo mutants. Genome editing techniques such as ZFNs (zinc finger nucleases), TALENs (transcription activator-like effector nucleases), the CRISPR (clustered regulatory interspaced short palindromic repeats)/Cas9 system, and the Tol2 transposon system have made zebrafish transgenesis very efficient. In combination with the fluorescent protein technology, the large number of transgenic lines allow for labeling of various subcellular structures, cells, tissues, and organs as well as for spatiotemporally reporting of the activity of specific signaling pathways $[56,104]$. The structural and functional visualization in vivo is very important to establish the correlation between genotype and phenotype. Their optical clarity and advantages in genetic engineering make zebrafish a model of choice when it comes to applying optical techniques involving fluorescent protein and optogenetic technologies. Technology advancements in this field allow new ways to visualize, quantify, and perturb developmental dynamics of the cardiovascular system. Recent application of light-sheet imaging enables the recapitulation and quantification of developmental cardiovascular mechanics $[112,113]$ that will further facilitate the study of mechanotransduction on cardiovascular development and homeostasis in the zebrafish model.

The general strengths of utilizing zebrafish for biomedical research are well known. Despite being phylogenetically distant from humans, zebrafish have remarkable similarities in genetics and electrophysiology. In the last 2 decades, a full array of genetic manipulation techniques have been developed and deployed in zebrafish. The critical mechanosensitive signaling pathways in cardiovascular development and physiology previously studied in mammals have been recapitulated in zebrafish. Studies in the zebrafish model further enhance our knowledge on the importance of mechanotransduction in cardiovascu- lar development and homeostasis. The application of genomic, transcriptomic, proteomic, and metabolomic analyses in the zebrafish provides a well-characterized platform for the exploration of many biological processes. Furthermore, the zebrafish model allows high throughput genetic and chemogenomic screening based on critical pathways, making it particularly attractive for therapeutic applications in human diseases.

\section{Acknowledgments}

Dr. Juhyun Lee and Dr. Nelson Jen assisted in the figure preparation.

\section{Statement of Ethics}

The authors have no ethical conflicts to disclose.

\section{Disclosure Statement}

The authors have no conflicts of interest to declare.

\section{Funding Sources}

The work was supported by the Startup Research Fund of the Shenzhen Technology University (R.L.) and the National Institutes of Health (R01HL111437 and R01HL129727 to T.K.H.).

\section{Author Contributions}

R.L. conceived, wrote, and revised the manuscript. K.I.B., C.C.C., and B.Z. wrote and revised the manuscript. T.K,H. conceived and revised the manuscript.

\section{References}

1 Lozano R, Naghavi M, Foreman K, Lim S, Shibuya K, Aboyans V, et al. Global and regional mortality from 235 causes of death for 20 age groups in 1990 and 2010: a systematic analysis for the Global Burden of Disease Study 2010. Lancet. 2012 Dec; 380(9859):2095-128. Erratum in: Lancet. 2013 Feb;381(9867):628. AlMazroa, Mohammad A [added]; Memish, Ziad A [added].

2 Genge CE, Lin E, Lee L, Sheng X, Rayani K, Gunawan $\mathrm{M}$, et al. The Zebrafish Heart as a
Model of Mammalian Cardiac Function. Rev Physiol Biochem Pharmacol. 2016;171:99136.

3 MacRae CA, Peterson RT. Zebrafish as tools for drug discovery. Nat Rev Drug Discov. 2015 Oct;14(10):721-31.

4 Santoriello C, Zon LI. Hooked! Modeling human disease in zebrafish. J Clin Invest. 2012 Jul;122(7):2337-43.

5 Vornanen M, Hassinen M. Zebrafish heart as a model for human cardiac electrophysiology. Channels (Austin). 2016;10(2):101-10.
6 Bakkers J. Zebrafish as a model to study cardiac development and human cardiac disease. Cardiovasc Res. 2011 Jul;91(2):279-88.

7 Guyon JR, Steffen LS, Howell MH, Pusack TJ, Lawrence C, Kunkel LM. Modeling human muscle disease in zebrafish. Biochim Biophys Acta. 2007 Feb;1772(2):205-15.

8 Auer TO, Duroure K, De Cian A, Concordet JP, Del Bene F. Highly efficient CRISPR/ Cas9-mediated knock-in in zebrafish by homology-independent DNA repair. Genome Res. 2014 Jan;24(1):142-53. 
9 Hoshijima K, Jurynec MJ, Grunwald DJ. Precise Editing of the Zebrafish Genome Made Simple and Efficient. Dev Cell. 2016 Mar; 36(6):654-67.

10 Kimura Y, Hisano Y, Kawahara A, Higashijima S. Efficient generation of knock-in transgenic zebrafish carrying reporter/driver genes by CRISPR/Cas9-mediated genome engineering. Sci Rep. 2014 Oct;4(1):6545.

11 Givens C, Tzima E. Endothelial Mechanosignaling: Does One Sensor Fit All? Antioxid Redox Signal. 2016 Sep;25(7):373-88.

12 Deng Q, Huo Y, Luo J. Endothelial mechanosensors: the gatekeepers of vascular homeostasis and adaptation under mechanical stress. Sci China Life Sci. 2014 Aug;57(8): $755-62$.

13 AbouAlaiwi WA, Takahashi M, Mell BR Jones TJ, Ratnam S, Kolb RJ, et al. Ciliary polycystin-2 is a mechanosensitive calcium channel involved in nitric oxide signaling cascades. Circ Res. 2009 Apr; 104(7):860-9.

14 Chachisvilis M, Zhang YL, Frangos JA. G protein-coupled receptors sense fluid shear stress in endothelial cells. Proc Natl Acad Sci USA. 2006 Oct; 103(42):15463-8

15 Jung B, Obinata H, Galvani S, Mendelson K, Ding BS, Skoura A, et al. Flow-regulated endothelial S1P receptor-1 signaling sustains vascular development. Dev Cell. 2012 Sep; 23(3):600-10.

16 Gulino-Debrac D. Mechanotransduction at the basis of endothelial barrier function. Tissue Barriers. 2013 Apr;1(2):e24180.

17 Tzima E, del Pozo MA, Shattil SJ, Chien S, Schwartz MA. Activation of integrins in endothelial cells by fluid shear stress mediates Rho-dependent cytoskeletal alignment. EMBO J. 2001 Sep;20(17):4639-47.

18 Yang B, Radel C, Hughes D, Kelemen S, Rizzo V. p190 RhoGTPase-activating protein links the $\beta 1$ integrin/caveolin-1 mechanosignaling complex to RhoA and actin remodeling. Arterioscler Thromb Vasc Biol. 2011 Feb;31(2):376-83.

19 Shyy JY, Chien S. Role of integrins in endothelial mechanosensing of shear stress. Circ Res. 2002 Nov;91(9):769-75.

20 Tzima E. Role of small GTPases in endothelial cytoskeletal dynamics and the shear stress response. Circ Res. 2006 Feb;98(2):176-85.

21 Tzima E, Del Pozo MA, Kiosses WB, Mohamed SA, Li S, Chien S, et al. Activation of Racl by shear stress in endothelial cells mediates both cytoskeletal reorganization and effects on gene expression. EMBO J. 2002 Dec 21(24):6791-800.

22 Li S, Chen BP, Azuma N, Hu YL, Wu SZ, Sumpio BE, et al. Distinct roles for the small GTPases Cdc42 and Rho in endothelial responses to shear stress. J Clin Invest. 1999 Apr;103(8):1141-50.

23 Tzima E, Kiosses WB, del Pozo MA, Schwartz MA. Localized cdc42 activation, detected using a novel assay, mediates microtubule organizing center positioning in endothelial cells in response to fluid shear stress. J Biol Chem. 2003 Aug;278(33):31020-3.
24 Birukov KG. Small GTPases in mechanosensitive regulation of endothelial barrier. $\mathrm{Mi}$ crovasc Res. 2009 Jan;77(1):46-52.

25 Zhou J, Li YS, Chien S. Shear stress-initiated signaling and its regulation of endothelial function. Arterioscler Thromb Vasc Biol. 2014 Oct;34(10):2191-8.

26 Yamamoto K, Sokabe T, Watabe T, Miyazono $\mathrm{K}$, Yamashita JK, Obi S, et al. Fluid shear stress induces differentiation of Flk-1-positive embryonic stem cells into vascular endothelial cells in vitro. Am J Physiol Heart Circ Physiol. 2005 Apr;288(4):H1915-24

27 Huang H, Nakayama Y, Qin K, Yamamoto K, Ando J, Yamashita J, et al. Differentiation from embryonic stem cells to vascular wall cells under in vitro pulsatile flow loading. J Artif Organs. 2005;8(2):110-8.

28 Wang H, Riha GM, Yan S, Li M, Chai H, Yang $\mathrm{H}$, et al. Shear stress induces endothelial differentiation from a murine embryonic mesenchymal progenitor cell line. Arterioscler Thromb Vasc Biol. 2005 Sep;25(9):1817-23.

29 Obi S, Yamamoto K, Shimizu N, Kumagaya S, Masumura T, Sokabe T, et al. Fluid shear stress induces arterial differentiation of endothelial progenitor cells. J Appl Physiol (1985). 2009 Jan;106(1):203-11.

30 Toh YC, Voldman J. Fluid shear stress primes mouse embryonic stem cells for differentiation in a self-renewing environment via heparan sulfate proteoglycans transduction. FASEB J. 2011 Apr;25(4):1208-17.

31 Krebs LT, Xue Y, Norton CR, Shutter JR, Maguire M, Sundberg JP, et al. Notch signaling is essential for vascular morphogenesis in mice. Genes Dev. 2000 Jun;14(11):1343-52.

32 Joutel A, Corpechot C, Ducros A, Vahedi K, Chabriat H, Mouton P, et al. Notch3 mutations in CADASIL, a hereditary adult-onset condition causing stroke and dementia. $\mathrm{Na}$ ture. 1996 Oct;383(6602):707-10.

33 Artavanis-Tsakonas S, Rand MD, Lake RJ. Notch signaling: cell fate control and signal integration in development. Science. 1999 Apr;284(5415):770-6.

34 Mack JJ, Mosqueiro TS, Archer BJ, Jones WM, Sunshine H, Faas GC, et al. NOTCH1 is a mechanosensor in adult arteries. Nat Commun. 2017 Nov;8(1):1620.

35 Masumura T, Yamamoto K, Shimizu N, Obi $\mathrm{S}$, Ando J. Shear stress increases expression of the arterial endothelial marker ephrinB2 in murine ES cells via the VEGF-Notch signaling pathways. Arterioscler Thromb Vasc Biol. 2009 Dec;29(12):2125-31.

36 Hove JR, Köster RW, Forouhar AS, AcevedoBolton G, Fraser SE, Gharib M. Intracardiac fluid forces are an essential epigenetic factor for embryonic cardiogenesis. Nature. 2003 Jan;421(6919):172-7.

37 Baek KI, Packard RR, Hsu JJ, Saffari A, Ma Z, Luu AP, et al. Ultrafine Particle Exposure Reveals the Importance of FOXO1/Notch Activation Complex for Vascular Regeneration. Antioxid Redox Signal. 2018 May;28(13): 1209-23.
38 Balciunaite E, Jones S, Toker A, Kazlauskas A. PDGF initiates two distinct phases of protein kinase $\mathrm{C}$ activity that make unequal contributions to the G0 to S transition. Curr Biol. 2000 Mar;10(5):261-7.

39 Racke FK, Wang D, Zaidi Z, Kelley J, Visvader J, Soh JW, et al. A potential role for protein kinase $\mathrm{C}$-epsilon in regulating megakaryocytic lineage commitment. J Biol Chem. 2001 Jan;276(1):522-8.

40 Mackay HJ, Twelves CJ. Targeting the protein kinase C family: are we there yet? Nat Rev Cancer. 2007 Jul;7(7):554-62.

41 Yamamura S, Nelson PR, Kent KC. Role of protein kinase $\mathrm{C}$ in attachment, spreading, and migration of human endothelial cells. J Surg Res. 1996 Jun;63(1):349-54.

42 Horowitz A, Clément-Chomienne O, Walsh MP, Morgan KG. Epsilon-isoenzyme of protein kinase $\mathrm{C}$ induces a $\mathrm{Ca}(2+)$-independent contraction in vascular smooth muscle. Am J Physiol. 1996 Aug;271(2 Pt 1):C589-94.

43 Rask-Madsen C, King GL. Differential regulation of VEGF signaling by PKC-alpha and PKC-epsilon in endothelial cells. Arterioscler Thromb Vasc Biol. 2008 May;28(5):919-24.

44 Carracedo S, Sacher F, Brandes G, Braun U, Leitges M. Redundant role of protein kinase $\mathrm{C}$ delta and epsilon during mouse embryonic development. PLoS One. 2014 Aug;9(8): e103686.

45 Koh W, Sachidanandam K, Stratman AN, Sacharidou A, Mayo AM, Murphy EA, et al. Formation of endothelial lumens requires a coordinated PKCepsilon-, Src-, Pak- and Rafkinase-dependent signaling cascade downstream of Cdc42 activation. J Cell Sci. 2009 Jun;122(Pt 11):1812-22.

46 Wang S, Iring A, Strilic B, Albarrán Juárez J, Kaur H, Troidl K, et al. P2 $\mathrm{Y}_{2}$ and $\mathrm{Gq} / \mathrm{G}_{11}$ control blood pressure by mediating endothelial mechanotransduction. J Clin Invest. 2015 Aug; 125(8):3077-86.

47 Jones R, Baker MB, Weber M, Harrison DG, Bao G, Searles CD. Molecular beacons can assess changes in expression and $3^{\prime}$-polyadenylation of human eNOS mRNA. Am J Physiol Cell Physiol. 2009 Mar;296(3):C498-504.

48 Inagaki K, Begley R, Ikeno F, Mochly-Rosen D. Cardioprotection by epsilon-protein kinase $\mathrm{C}$ activation from ischemia: continuous delivery and antiarrhythmic effect of an epsilon-protein kinase $\mathrm{C}$-activating peptide. Circulation. 2005 Jan;111(1):44-50.

49 Budas G, Costa HM Jr, Ferreira JC, Teixeira da Silva Ferreira A, Perales J, Krieger JE, et al. Identification of $\varepsilon \mathrm{PKC}$ targets during cardiac ischemic injury. Circ J. 2012;76(6):1476-85.

50 Cross HR, Murphy E, Bolli R, Ping P, Steenbergen C. Expression of activated PKC epsilon (PKC epsilon) protects the ischemic heart, without attenuating ischemic $\mathrm{H}(+)$ production. J Mol Cell Cardiol. 2002 Mar;34(3): 361-7. 
51 Edmondson RD, Vondriska TM, Biederman KJ, Zhang J, Jones RC, Zheng Y, et al. Protein kinase $\mathrm{C}$ epsilon signaling complexes include metabolism- and transcription/translationrelated proteins: complimentary separation techniques with LC/MS/MS. Mol Cell Proteomics. 2002 Jun;1(6):421-33.

52 Mayr M, Liem D, Zhang J, Li X, Avliyakulov NK, Yang JI, et al. Proteomic and metabolomic analysis of cardioprotection: interplay between protein kinase $\mathrm{C}$ epsilon and delta in regulating glucose metabolism of murine hearts. J Mol Cell Cardiol. 2009 Feb;46(2): 268-77.

53 De Bock K, Georgiadou M, Carmeliet P. Role of endothelial cell metabolism in vessel sprouting. Cell Metab. 2013 Nov;18(5):63447.

54 Suárez J, Rubio R. Regulation of glycolytic flux by coronary flow in guinea pig heart. Role of vascular endothelial cell glycocalyx. Am J Physiol. 1991 Dec;261(6 Pt 2):H1994-2000.

55 De Bock K, Georgiadou M, Schoors S, Kuchnio A, Wong BW, Cantelmo AR, et al. Role of PFKFB3-driven glycolysis in vessel sprouting. Cell. 2013 Aug;154(3):651-63.

56 Baek KI, Li R, Jen N, Choi H, Kaboodrangi A, Ping P, et al. Flow-Responsive Vascular Endothelial Growth Factor Receptor-Protein Kinase C Isoform Epsilon Signaling Mediates Glycolytic Metabolites for Vascular Repair. Antioxid Redox Signal. 2018 Jan;28(1):31-43.

57 Tressel SL, Huang RP, Tomsen N, Jo H. Laminar shear inhibits tubule formation and migration of endothelial cells by an angiopoietin-2 dependent mechanism. Arterioscler Thromb Vasc Biol. 2007 Oct;27(10):2150-6.

58 Ahmed A, Fujisawa T, Niu XL, Ahmad S, AlAni B, Chudasama K, et al. Angiopoietin-2 confers Atheroprotection in apoE-/- mice by inhibiting LDL oxidation via nitric oxide. Circ Res. 2009 Jun;104(12):1333-6.

59 Ahmed A, Fujisawa T. Multiple roles of angiopoietins in atherogenesis. Curr Opin Lipidol. 2011 Oct;22(5):380-5.

60 Thomas M, Augustin HG. The role of the Angiopoietins in vascular morphogenesis. Angiogenesis. 2009;12(2):125-37.

61 Felcht M, Luck R, Schering A, Seidel P, Srivastava $\mathrm{K}, \mathrm{Hu}$ J, et al. Angiopoietin-2 differentially regulates angiogenesis through TIE2 and integrin signaling. J Clin Invest. 2012 Jun; 122(6):1991-2005.

62 Korff T, Ernst E, Nobiling R, Feldner A, Reiss $\mathrm{Y}$, Plate $\mathrm{KH}$, et al. Angiopoietin-1 mediates inhibition of hypertension-induced release of angiopoietin-2 from endothelial cells. Cardiovasc Res. 2012 Jun;94(3):510-8.

63 Li R, Beebe T, Jen N, Yu F, Takabe W, Harrison $\mathrm{M}$, et al. Shear stress-activated Wnt-angiopoietin-2 signaling recapitulates vascular repair in zebrafish embryos. Arterioscler Thromb Vasc Biol. 2014 Oct;34(10):2268-75.
64 Tressel SL, Kim H, Ni CW, Chang K, Velasquez-Castano JC, Taylor WR, et al. Angiopoietin-2 stimulates blood flow recovery after femoral artery occlusion by inducing inflammation and arteriogenesis. Arterioscler Thromb Vasc Biol. 2008 Nov;28(11):198995.

65 Logan CY, Nusse R. The Wnt signaling pathway in development and disease. Annu Rev Cell Dev Biol. 2004;20(1):781-810.

66 Chen H, Shi S, Acosta L, Li W, Lu J, Bao S, et al. BMP10 is essential for maintaining cardiac growth during murine cardiogenesis. Development. 2004 May;131(9):2219-31.

67 Li J, Hou B, Tumova S, Muraki K, Bruns A, Ludlow MJ, et al. Piezol integration of vascular architecture with physiological force. $\mathrm{Na}$ ture. 2014 Nov;515(7526):279-82.

68 Banjo T, Grajcarek J, Yoshino D, Osada H, Miyasaka KY, Kida YS, et al. Haemodynamically dependent valvulogenesis of zebrafish heart is mediated by flow-dependent expression of miR-21. Nat Commun. 2013;4(1): 1978.

69 Ten Dijke P, Egorova AD, Goumans MJ, Poelmann RE, Hierck BP. TGF- $\beta$ signaling in endothelial-to-mesenchymal transition: the role of shear stress and primary cilia. Sci Signal. 2012 Feb;5(212):pt2.

70 Santhanakrishnan A, Miller LA. Fluid dynamics of heart development. Cell Biochem Biophys. 2011 Sep;61(1):1-22.

71 Lucitti JL, Jones EA, Huang C, Chen J, Fraser SE, Dickinson ME. Vascular remodeling of the mouse yolk sac requires hemodynamic force. Development. 2007 Sep;134(18):3317-26.

72 Peshkovsky C, Totong R, Yelon D. Dependence of cardiac trabeculation on neuregulin signaling and blood flow in zebrafish. Dev Dyn. 2011 Feb;240(2):446-56.

73 Zhang W, Chen H, Qu X, Chang CP, Shou W. Molecular mechanism of ventricular trabeculation/compaction and the pathogenesis of the left ventricular noncompaction cardiomyopathy (LVNC). Am J Med Genet C Semin Med Genet. 2013 Aug;163C(3):144-56.

74 Nugent AW, Daubeney PE, Chondros P, Carlin JB, Cheung M, Wilkinson LC, et al.; National Australian Childhood Cardiomyopathy Study. The epidemiology of childhood cardiomyopathy in Australia. N Engl J Med. 2003 Apr;348(17):1639-46.

75 D’Amato G, Luxán G, del Monte-Nieto G, Martínez-Poveda B, Torroja C, Walter W, et al. Sequential Notch activation regulates ventricular chamber development. Nat Cell Biol. 2016 Jan;18(1):7-20.

76 Grego-Bessa J, Luna-Zurita L, del Monte G, Bolós V, Melgar P, Arandilla A, et al. Notch signaling is essential for ventricular chamber development. Dev Cell. 2007 Mar;12(3):41529.

77 Gordon WR, Zimmerman B, He L, Miles LJ, Huang J, Tiyanont K, et al. Mechanical Allostery: Evidence for a Force Requirement in the Proteolytic Activation of Notch. Dev Cell. 2015 Jun;33(6):729-36.
78 Lee J, Fei P, Packard RR, Kang H, Xu H, Baek KI, et al. 4-Dimensional light-sheet microscopy to elucidate shear stress modulation of cardiac trabeculation. J Clin Invest. 2016 May; 126(5):1679-90.

79 Vermot J, Forouhar AS, Liebling M, Wu D, Plummer D, Gharib M, et al. Reversing blood flows act through klf2a to ensure normal valvulogenesis in the developing heart. PLoS Biol. 2009 Nov;7(11):e1000246.

80 Galloway JL, Wingert RA, Thisse C, Thisse B, Zon LI. Loss of gata1 but not gata2 converts erythropoiesis to myelopoiesis in zebrafish embryos. Dev Cell. 2005 Jan;8(1):109-16.

81 Arnaout R, Ferrer T, Huisken J, Spitzer K, Stainier DY, Tristani-Firouzi M, et al. Zebrafish model for human long QT syndrome. Proc Natl Acad Sci U S A. 2007 Jul;104(27): 11316-21.

82 Chi NC, Shaw RM, Jungblut B, Huisken J, Ferrer T, Arnaout R, et al. Genetic and physiologic dissection of the vertebrate cardiac conduction system. PLoS Biol. 2008 May; 6(5):e109.

83 Berdougo E, Coleman H, Lee DH, Stainier DY, Yelon D. Mutation of weak atrium/atrial myosin heavy chain disrupts atrial function and influences ventricular morphogenesis in zebrafish. Development. 2003 Dec;130(24): 6121-9.

84 Liao W, Bisgrove BW, Sawyer H, Hug B, Bell $\mathrm{B}$, Peters $\mathrm{K}$, et al. The zebrafish gene cloche acts upstream of a flk-1 homologue to regulate endothelial cell differentiation. Development. 1997 Jan;124(2):381-9.

85 Stainier DY, Weinstein BM, Detrich HW 3rd, Zon LI, Fishman MC. Cloche, an early acting zebrafish gene, is required by both the endothelial and hematopoietic lineages. Development. 1995 Oct;121(10):3141-50.

86 High FA, Epstein JA. The multifaceted role of Notch in cardiac development and disease. Nat Rev Genet. 2008 Jan;9(1):49-61.

87 Hedhli N, Huang Q, Kalinowski A, Palmeri $\mathrm{M}, \mathrm{Hu} \mathrm{X}$, Russell RR, et al. Endotheliumderived neuregulin protects the heart against ischemic injury. Circulation. 2011 May; 123(20):2254-62.

88 Fang SJ, Wu XS, Han ZH, Zhang XX, Wang CM, Li XY, et al. Neuregulin-1 preconditioning protects the heart against ischemia/reperfusion injury through a PI3K/Akt-dependent mechanism. Chin Med J (Engl). 2010 Dec; 123(24):3597-604.

89 Liu J, Bressan M, Hassel D, Huisken J, Staudt D, Kikuchi K, et al. A dual role for ErbB2 signaling in cardiac trabeculation. Development. 2010 Nov; $137(22): 3867-75$.

90 Lee J, Fei P, Sevag Packard RR, Kang H, Xu H, Baek KI, et al. 4-Dimensional light-sheet microscopy to elucidate shear stress modulation of cardiac trabeculation. J Clin Invest. 2016 Aug;126(8):3158. 
91 Parkinson disease. Metablomics study reveals novel biomarkers for PD. Nat Rev Neurol. 2013;9:484.

92 Stroke: molecular markers could help predict stroke risk after TIA. Nat Rev Neurol. 2015;11(1):3.

93 Clyne M. Kidney cancer: metabolomics for targeted therapy. Nat Rev Urol. 2012 Jun; 9(7):355.

94 Dunn J, Qiu H, Kim S, Jjingo D, Hoffman R, Kim CW, et al. Flow-dependent epigenetic DNA methylation regulates endothelial gene expression and atherosclerosis. J Clin Invest. 2014 Jul;124(7):3187-99.

95 Frangos JA, McIntire LV, Eskin SG. Shear stress induced stimulation of mammalian cell metabolism. Biotechnol Bioeng. 1988 Oct;32(8):1053-60.

96 Hwang J, Ing MH, Salazar A, Lassègue B, Griendling K, Navab $M$, et al. Pulsatile versus oscillatory shear stress regulates NADPH oxidase subunit expression: implication for native LDL oxidation. Circ Res. 2003 Dec;93(12):1225-32.

97 Kluge MA, Fetterman JL, Vita JA. Mitochondria and endothelial function. Circ Res. 2013 Apr;112(8):1171-88.

98 Sun X, Feinberg MW. Regulation of endothelial cell metabolism: just go with the flow. Arterioscler Thromb Vasc Biol. 2015 Jan; 35(1):13-5.

99 Wang Y, Zang QS, Liu Z, Wu Q, Maass D, Dulan G, et al. Regulation of VEGF-induced endothelial cell migration by mitochondrial reactive oxygen species. Am J Physiol Cell Physiol. 2011 Sep;301(3):C695-704.
100 Neufer PD, Bamman MM, Muoio DM, Bouchard C, Cooper DM, Goodpaster $\mathrm{BH}$ et al. Understanding the Cellular and Molecular Mechanisms of Physical Activity-Induced Health Benefits. Cell Metab. 2015 Jul; 22(1):4-11.

101 Culic O, Gruwel ML, Schrader J. Energy turnover of vascular endothelial cells. Am J Physiol. 1997 Jul;273(1 Pt 1):C205-13.

102 Doddaballapur A, Michalik KM, Manavski Y, Lucas T, Houtkooper RH, You X, et al. Laminar shear stress inhibits endothelial cell metabolism via KLF2-mediated repression of PFKFB3. Arterioscler Thromb Vasc Biol. 2015 Jan;35(1):137-45.

103 Qin X, Tian J, Zhang P, Fan Y, Chen L, Guan $\mathrm{Y}$, et al. Laminar shear stress up-regulates the expression of stearoyl-CoA desaturase-1 in vascular endothelial cells. Cardiovasc Res. 2007 Jun;74(3):506-14.

104 Nakajima H, Yamamoto K, Agarwala S, Terai K, Fukui H, Fukuhara S, et al: Flow-Dependent Endothelial YAP Regulation Contributes to Vessel Maintenance. Dev Cell. 2017;40:523-536.e6.

105 Chan P, Han X, Zheng B, DeRan M, Yu J, Jarugumilli GK, et al. Autopalmitoylation of TEAD proteins regulates transcriptional output of the Hippo pathway. Nat Chem Biol. 2016 Apr;12(4):282-9.

106 Choi HJ, Zhang H, Park H, Choi KS, Lee HW, Agrawal V, et al. Yes-associated protein regulates endothelial cell contact-mediated expression of angiopoietin-2. Nat Commun. 2015 May;6(1):6943.
107 Lee J, Moghadam ME, Kung E, Cao H, Beebe T, Miller Y, et al. Moving domain computational fluid dynamics to interface with an embryonic model of cardiac morphogenesis. PLoS One. 2013 Aug;8(8):e72924.

108 Samsa LA, Givens C, Tzima E, Stainier DY, Qian L, Liu J. Cardiac contraction activates endocardial Notch signaling to modulate chamber maturation in zebrafish. Development. 2015 Dec;142(23):4080-91.

109 Han P, Bloomekatz J, Ren J, Zhang R, Grinstein JD, Zhao L, et al. Coordinating cardiomyocyte interactions to direct ventricular chamber morphogenesis. Nature. 2016 Jun; 534(7609):700-4

110 Lee J, Vedula V, Baek KI, Chen J, Hsu JJ, Ding Y, et al. Spatial and temporal variations in hemodynamic forces initiate cardiac trabeculation. JCI Insight. 2018 Jul;3 (13):3.

111 Kettleborough RN, Busch-Nentwich EM, Harvey SA, Dooley CM, de Bruijn E, van Eeden $\mathrm{F}$, et al. A systematic genome-wide analysis of zebrafish protein-coding gene function. Nature. 2013 Apr;496(7446): 494-7.

112 Ding Y, Abiri A, Abiri P, Li S, Chang CC, Baek KI, et al. Integrating light-sheet imaging with virtual reality to recapitulate developmental cardiac mechanics. JCI Insight. 2017 Nov;2(22):2.

113 Vedula V, Lee J, Xu H, Kuo CJ, Hsiai TK, Marsden AL. A method to quantify mechanobiologic forces during zebrafish cardiac development using 4-D light sheet imaging and computational modeling. PLoS Comput Biol. 2017 Oct;13(10):e1005828. 